\title{
Inhibition of lipid autoxidation by vegetable waxes
}

\section{Valoppi, Fabio}

2020-07-01

\section{Valoppi , F , Haman , N , Ferrentino , G \& Scampicchio , M 2020 , ' Inhibition of lipid} autoxidation by vegetable waxes ' , Food \& Function , vol. 11 , no. 7 , pp. 6215-6225 . https://doi.org/10.1039/d0fo01

http://hdl.handle.net/10138/318551

https://doi.org/10.1039/d0fo01022g

acceptedVersion

Downloaded from Helda, University of Helsinki institutional repository.

This is an electronic reprint of the original article.

This reprint may differ from the original in pagination and typographic detail.

Please cite the original version. 


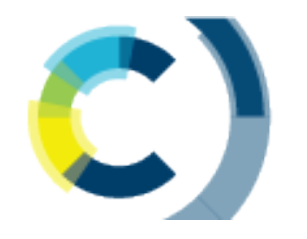

\section{Food \& Function}

\section{Inhibition of Lipid Autoxidation by Vegetable Waxes}

\begin{tabular}{|c|c|}
\hline Journal: & Food \& Function \\
\hline Manuscript ID & FO-ART-04-2020-001022.R2 \\
\hline Article Type: & Paper \\
\hline $\begin{array}{r}\text { Date Submitted by the } \\
\text { Author: }\end{array}$ & 24-Jun-2020 \\
\hline Complete List of Authors: & $\begin{array}{l}\text { Valoppi, Fabio; University of Helsinki, Food and Nutrition } \\
\text { Haman, Nabil; Free University of Bozen-Bolzano } \\
\text { Ferrentino, Giovanna; Free University of Bozen-Bolzano, } \\
\text { scampicchio, matteo; Free University of Bozen-Bolzano }\end{array}$ \\
\hline
\end{tabular}

\section{SCHOLARONE ${ }^{m}$ Manuscripts}




\section{Inhibition of Lipid Autoxidation by Vegetable Waxes}

Fabio Valoppi ${ }^{\mathrm{a}, \mathrm{b}, \#, \text { Nabil Haman }}$, \#, Giovanna Ferrentino ${ }^{\mathrm{c},}{ }^{*}$, Matteo Scampicchio ${ }^{\mathrm{c}}$

${ }^{a}$ Department of Food and Nutrition, P.O. Box 66 (Agnes Sjöbergin katu 2), University of Helsinki, FI-0014 Helsinki, Finland

${ }^{\mathrm{b}}$ Helsinki Institute of Sustainability Science, Faculty of Agriculture and Forestry, University of Helsinki, Finland

${ }^{\mathrm{c}}$ Free University of Bolzano, Faculty of Science and Technology, Piazza Università 1, 39100 Bolzano, Italy

*Corresponding author:

e-mail: giovanna.ferrentino@unibz.it, phone: +39 0471 017692, address: piazza Università 5, 39100 Bozen-Bolzano, Italy.

\#These authors equally contributed. 


\begin{abstract}
This study aims to evaluate the effect of vegetable waxes on the kinetic of lipid oxidation of linseed oil. Apples and orange waxes were obtained by supercritical carbon dioxide. The capacity of waxes to inhibit or retard the oxidation of linseed oil was determined by isothermal calorimetry at $298 \mathrm{~K}$. The results show that waxes were able to slow down linseed oil autoxidation, with apple waxes being more active than orange waxes. However, such activity was visible only at relatively high concentrations ( $>1 \%$ of waxes), greatly higher than the concentration used with radical chain breakers like BHT $(0.2 \%)$. The inhibition activity was explained by considering three different mechanisms, respectively, (1) residual polyphenol content in the wax, (2) high termination rate of the radical chain process, (3) physical hindrance of the oxidation process by change of viscosity. All these mechanisms were possible, although the latter seemed to be the most important. Finally, significance of waxes to inhibit lipid autoxidation was determined by testing their inhibition activity in cooperation with primary antioxidants. Mixture of waxes with BHA, ethoxyquin and $\alpha-$ tocopherol showed higher rate of inhibition than when present individually. This suggested a strong cooperative radical scavenging activity, whose beneficial effect might pave the way to the formulation of novel functional ingredients.
\end{abstract}

Keywords: linseed oil; vegetable waxes; oxidative stability; isothermal calorimetry; supercritical fluid extraction; fruit by-products. 


\section{Introduction}

Waxes are esters of long-chain alcohols and fatty acids. This definition comprises a broad class of lipid substances, that may include other functional groups, such as ketones, aldehydes and aromatic compounds ${ }^{1,2}$. Among the different types of waxes, those derived from plants have received an increasing interest because of the growing demand of biomaterials. Based on their chemical composition, waxes can find important applications as thickeners, moisturizers, and preservatives in food, cosmetic, and pharmaceutical products ${ }^{3}$. Recent works claim that vegetable waxes possess also antioxidant, antimicrobial and antifungal functionalities ${ }^{4}$.

Among these functionalities, the capacity of waxes to delay lipid oxidation has been recently debated. Some works explain such capacity with the residual presence of aromatic compounds, such as flavonoids, sterols and phenolics, from the extraction process ${ }^{4-6}$. Other works observed a higher oxidative stability in products containing waxes ${ }^{7}$ and explained it with the increase of viscosity, which can indirectly slow down the rate of oxidation of the resulting mixture ${ }^{8-10}$. For instance, a work of Yilmaz and Ogutcu ${ }^{7}$ showed that mixing beeswax with hazelnut oil led to a product very stable against oxidation during storage. Later, Ogutcu et al. demonstrated that fish oil prepared with sunflower wax were more oxidatively stable than the same sample prepared with saturated monoglycerides ${ }^{11}$. Yi et al. showed that the oxidative stability of oils prepared with carnauba wax was higher compared to bulk oils ${ }^{12}$.

However, all the aforementioned studies focused on the evidence that vegetable waxes may exert an antioxidant activity but few of them deeply described the complex mechanism ruling the activity of waxes in oil. 
As far as it was observed so far, the final effect of wax on oils can be ruled by the combination of several factors such as the antioxidant activity of impurities in waxes or the kinetic hindrance on oxygen diffusion exerted by the crystal network.

Some works also reported that waxes may be responsible for some prooxidant activity or no activity against oil oxidation ${ }^{7,13-15}$. Overall, the mechanism ruling out the functional activity of waxes towards lipid oxidation processes is unclear.

A further interesting aspect is linked to the possible use of vegetable waxes combined with primary antioxidants to study their action on the oil oxidative stability. Currently, little and fragmented information is available in the literature. To our knowledge, just one study has been recently published by Martinović et al. who studied the effect of the addition of sinapic acid and its derivaties (syringic acid, syringaldehyde, sinapine, 4-vinylsyringol, and ethyl, propyl and butyl sinapate) in oil systems containing vegetable waxes ${ }^{14}$. They demonstrated that the use of sinapic acid in sunflower oil with $5 \%$ of beeswax decreased the peroxidation of the oil entrapped in the crystalline structures. However, they also suggested the need to perform more studies to further elucidate the synergistic actions occurring between primary antioxidants and vegetable waxes. These investigations could open new perspectives for the application of vegetable waxes in oilbased products in order to improve their use as ingredients with antioxidant activity. Accordingly, the aim of this work was to understand the effect of vegetable waxes on the oxidation kinetic of lipid oxidation by isothermal calorimetry. Previous studies showed its capacity to monitor food reactions. Lipid oxidation is an exothermic phenomenon that can be followed by isothermal calorimetry. Measurements can be performed under a static air atmosphere. Isothermal experiments allow induction times of oxidation to be determined. Isothermal calorimetry can, 
therefore, be used to assess the oxidative stability of lipids or the efficiency of food antioxidants in bulk lipids.

Recently, isothermal calorimetry was applied to study the inhibition of linoleic acid oxidation in presence of natural and synthetic antioxidants. The results showed a correlation between the heat flow signals and the evolution of volatile compounds developed during the oxidation ${ }^{16}$. Further studies have been also carried out applying the technique to follow the autoxidation reaction of different oils such as fish, lard, corn, and linseed oil ${ }^{17}$ or to assess the antioxidant and prooxidant activity of extracts from spent coffee powder ${ }^{18}$.

In this study, apples and orange waxes (extracted by supercritical carbon dioxide) and a refined sunflower wax were used. Their effects on the inhibition of linseed oil oxidation by isothermal calorimetry were investigated in terms of (i) wax concentration and (ii) type, (iii) cooling rate, and (iv) interaction between waxes and primary antioxidants. Overall, this work provides some useful insights on the potential use of vegetable waxes as retarder of lipid oxidation.

\section{Materials and methods}

\subsection{Materials}

Linseed oil was purchased from Sigma Aldrich (CAS number 8001-26-1, Milano, Italy). The composition of the oil was: $4.2 \%$ stearic acid, $4.8 \%$ palmitic acid, $19.5 \%$ oleic acid, $18.1 \%$ linoleic acid, $51.3 \%$ linolenic acid, $2.1 \%$ other minor compounds. Purified sunflower wax was kindly donated by L.C.M. S.p.A. (Sesto San Giovanni, Milan, Italy) and was composed of wax esters with a chain length (CL) between 42 and 56 carbon atoms. The composition of the wax was: $1.92 \%$ C42, $0.45 \%$ C43; $17.89 \%$ C44, $1.80 \%$ C45, $25.89 \%$ C46, $1.61 \%$ C47, 18.81 C48, 1.16 \% C49, $12.69 \%$ C50, 1.86 \% C51, $8.35 \%$ C52, $0.68 \%$ C53, $4.26 \%$ C54, $0.33 \%$ C55, $2.30 \%$ 
C56. Waxes did not contain flavonols or carotenoids. All reagents used in this study were analytical grade (>96\%, HPLC) and purchased from Sigma-Aldrich (Milan, Italy).

\subsection{Preparation of apple and orange peels}

Apple and orange peels were purchased from a local market. The fruits were peeled and the peels were characterized by an initial moisture of $88.6 \pm 2.4 \%$ and $65.5 \pm 1.5 \%$ (Sartorious MA160, Torino, Italy) and water activity of $0.93 \pm 0.01$ and $0.88 \pm 0.01$ (AquaLab, Steroglass, Perugia, Italy), respectively. They were freeze dried (Epsilon 2-6D LSC plus freeze-drier, Martin Christ, Osterode am Harz, Germany) setting the freezing at $-18^{\circ} \mathrm{C}$ for $4 \mathrm{~h}$, the primary drying for $20 \mathrm{~h}$ at a temperature of $35^{\circ} \mathrm{C}$ and a vacuum of $1.01 \mathrm{mbar}$, and then the secondary drying phase for $6 \mathrm{~h}$ at a temperature of $40^{\circ} \mathrm{C}$ and a vacuum of 0.05 mbar. The dried samples reached a moisture content of $20.5 \pm 1.7 \%$ for apple peels and $10.2 \pm 1.5 \%$ for orange peels with water activity equal to 0.15 \pm 0.02 . Both dried peels were ground with a laboratory hammer mill (Perten Instruments, Hägersten Sweden) reaching a final particle size of $0.8 \mathrm{~mm}$.

\subsection{Extraction of waxes by supercritical carbon dioxide}

Extraction from apple and orange peels powders was carried out by supercritical fluid extraction at pilot scale (Superfluidi s.r.1., Padova, Italy). The high pressure vessel (1 L volume) contained an extraction basket of $800 \mathrm{~mL}$, closed with porous stainless steel mesh filters. The $\mathrm{CO}_{2}$ was pressurized by a high pressure diaphragm pump (Lewa LDC-M-9XXV1, Milano, Italy) with jacketed heads for cooling. The system was equipped with a high pressure tank for $\mathrm{CO}_{2}$ storage to be recirculated and further reused. The flow rate of $\mathrm{CO}_{2}$ was set to $2 \mathrm{~L} / \mathrm{h}$. in the extracts. This value was selected in order to ensure a long residence time of the solvent and a prolonged contact with the sample. About $40 \pm 1 \mathrm{~g}$ of dried apple or orange powder, with the addition of $5 \%(\mathrm{w} / \mathrm{w})$ of 
ethanol, was filled inside the extractor. The extraction time was fixed to $120 \mathrm{~min}$. During the first 60 min, a static extraction was performed followed by a dynamic extraction by flushing $\mathrm{CO}_{2}$ through the samples for the remaining $60 \mathrm{~min}$. The optimum pressure and temperature were set at $30 \mathrm{MPa}$ and $323 \mathrm{~K}$ for apple and orange peels extraction. The conditions were chosen based on preliminary experiments performed to identify the process conditions to achieve the highest extraction yield. At the end of the extraction time, the extract was collected in amber flasks and weighted. After each extraction, the vessel and the tubes of the system were cleaned by washing them with $25 \mathrm{~mL}$ of ethanol. The cleaning procedure allowed to recover all the extracted residues from the samples. After the cleaning, the sample was collected in a $250 \mathrm{~mL}$ volumetric flask and the ethanol was removed and merged with the extract previously collected. The obtained samples from the extraction were first centrifuged and then the supernatants were removed. The extracts were flushed with nitrogen for $6 \mathrm{~h}$ to completely remove the remained ethanol. The extraction led to a yield of $4.2 \pm 0.5 \%(\mathrm{w} / \mathrm{w})$ for apple wax and $3.5 \pm 0.8 \%(\mathrm{w} / \mathrm{w})$ for orange wax. The yield was determined as ratio between the amount of extract and the amount of sample used for the extraction, on dry weight basis.

\subsection{Characterization of apple and orange waxes}

\subsubsection{Chemical composition of apple and orange waxes by GC-MS}

The obtained waxes from supercritical carbon dioxide were treated with bis-(trimethylsilyl)trifluoroucetamide with $1 \%$ trimethylchlorosilane (TMCS) and $100 \mu \mathrm{L}$ of toluene at $348 \mathrm{~K}$ for 30 min. After the evaporation under continuous $\mathrm{N}_{2}$ flush, the sample were re-dissolved in hexane for the analysis with an Agilent 6890/5973 GC/MS network gas system (Agilent Technologies) and a HP-5MS high-temperature capillary column $(30 \mathrm{~m} \times 0.25 \mathrm{~mm} \times 0.25 \mu \mathrm{m})$. Helium was used as the carrier gas at a flow rate of $1.0 \mathrm{~mL} / \mathrm{min}$. The analysis was carried oud with the following 
settings: the initial temperature of $343 \mathrm{~K}(1 \mathrm{~min})$ was ramped at $15 \mathrm{~K} \mathrm{~min}^{-1}$ to $493 \mathrm{~K}$; then increased to $553 \mathrm{~K}$ at $5 \mathrm{~K} \mathrm{~min}^{-1}$, and held at this temperature for $8 \mathrm{~min}$. The following other parameters were employed: ion source temperature, $503 \mathrm{~K}$; MS transfer line temperature, $563 \mathrm{~K}$; electron impact (EI), $70 \mathrm{eV} ; \mathrm{m}=\mathrm{z}$ range, 35-650. The mass spectra of the wax compounds were identified with the help of standard reference data and related literatures. The chemical composition of the extracted waxes is reported in Table 1.

\subsubsection{Total phenolic content}

The total phenolic content of apple and orange waxes was determined by the Folin-Ciocalteau method as described by Singleton and Rossi ${ }^{19}$. The samples $(300 \mathrm{mg})$ were diluted in $1.5 \mathrm{~mL}$ of ethanol and sonicated for $5 \mathrm{~min}$ at $298 \mathrm{~K}$ for three times. The ethanolic extracts after the sonication were collected and diluted in ethanol. For the assay the cuvettes were filled with $1.2 \mathrm{~mL}$ distilled water, and $40 \mu \mathrm{L}$ of extract, $300 \mu \mathrm{L}$ of $20 \%$ sodium carbonate solution and $100 \mu \mathrm{L}$ of FolinCiocalteau. Samples were held for $2 \mathrm{~h}$ in darkness and the absorbance was measured with a spectrophotometer (Cary 100 Series UV-Vis Spectrophotometer, Agilent Technologies, Italy) at $765 \mathrm{~nm}$. The total phenolic content was calculated according to a standard calibration curve using gallic acid as standard. The results were expressed as milligrams of gallic acid equivalent (GAE) per gram of wax (mg GAE/g of wax).

\subsection{Linseed oil with natural waxes preparation}

For the sample preparation, $1000 \pm 5 \mathrm{mg}$ of linseed oil was weighted in $10-\mathrm{mL}$ glass vials. To these oil samples, $3 \%(\mathrm{w} / \mathrm{w})$ of sunflower, apple peel or orange peel waxes were added respectively. The vials were saturated with nitrogen and hermetically sealed to perform the experiment in controlled atmosphere in absence of oxygen. The samples were placed in a temperature-controlled water bath at $353 \mathrm{~K}$ for $20 \mathrm{~min}$ and shaken every 2 min to mix the molten 
wax and linseed oil. The control, bulk linseed oil without wax, was also subjected to this procedure to undergo same oxidation during this step. Around $300 \pm 10 \mathrm{mg}$ of the molten system was then transferred into 4-mL glass ampoules which were hermetically sealed and cooled at room temperature using different cooling rates. The cooling rate was measured by putting a wire probe at the center of an extra sample in a vial. Two set of samples were cooled in three different water cooling bath with set temperatures of 298,288 and $278 \mathrm{~K}$, resulting in cooling rates of $0.6 \pm 0.1$, $1.0 \pm 0.2$, and $6.0 \pm 0.1 \mathrm{~K} \cdot \mathrm{min}^{-1}$, respectively. The other set of samples was cooled by immersing the sample vial in an ice bath, resulting in a cooling rate of $30.3 \pm 1.5 \mathrm{~K} \cdot \mathrm{min}^{-1}$.

\subsubsection{Addition of different waxes concentration to linseed oil}

To assess the effect of concentration, increasing amount of sunflower, apple or orange waxes were mixed with $1000 \pm 0.05 \mathrm{mg}$ of linseed oil to obtain concentrations of $1,2,3$, and $5 \% \mathrm{w} / \mathrm{w}$. The mixture was melted in a water bath at $353 \mathrm{~K}$ for $20 \mathrm{~min}$. Then, $300 \pm 10 \mathrm{mg}$ of the molten system was then transferred into 4-mL glass ampoules and cooled at room temperature at a rate equal to $6.0 \pm 0.2 \mathrm{~K} \cdot \mathrm{min}^{-1}$

\subsubsection{Addition of waxes and primary antioxidants to linseed oil}

To study the effect of the antioxidant addition, about $1000 \pm 0.05 \mathrm{mg}$ of linseed oil was enriched with $( \pm) \alpha$-tocopherol, BHA, or ethoxyquin to have a final concentration of $1 \mathrm{mM}$ of antioxidants in oil. To fasten the dissolution of the antioxidants, the enriched oils were sonicated for $6 \mathrm{~min}$ using an ultrasonic bath (Digital ultrasonic bath Mod. DU-06, ArgoLab, Modena, Italy). Subsequently, to these samples $1 \%(\mathrm{w} / \mathrm{w})$ of sunflower wax was added. After melting in the temperature-controlled water bath at $353 \mathrm{~K}$ for $20 \mathrm{~min}$, about $300 \pm 10 \mathrm{mg}$ of the molten system was then transferred into 4-mL glass ampoules and cooled at room temperature with a cooling rate equal to $6.0 \pm 0.2 \mathrm{~K} \cdot \mathrm{min}^{-1}$. After cooling, all the ampoules were left at room temperature for 10 
min. They were subsequently opened for 5 min to permit the air to diffuse inside and hermetically sealed again. Three replicates were used for each sample for the analyses.

\subsection{Oxidative stability by isothermal calorimetry}

The oxidative stability of samples containing different concentration and type of waxes and antioxidants in linseed oil was measured by isothermal calorimetry. For the calorimetric analysis, a micro-calorimetry thermostat (Thermal Activity Monitor, Model 421 TAM III, TA Instruments) was used. The thermostat was equipped with 24 micro-calorimetric channels, each of them positioned above each other as a twin calorimeter, and it was used to measure the heat flow signals. The micro-calorimeters were equipped with built-in metal reference specimens having a heat capacity approximately equal to that of an ampoule. In isothermal mode, the samples in the thermostat were maintained at a temperature of $298 \mathrm{~K}$ with an absolute accuracy of $\pm 0.00005 \mathrm{~K}$ (manufacturer's data). They were prepared as previously described. Then, around $300 \pm 10 \mathrm{mg}$ of the molten system was transferred into 4-mL glass ampoules which were hermetically sealed with silicone septa and cooled at room temperature. The control, bulk linseed oil, which underwent the same preparation procedure was also analyzed. The heat generated or absorbed was continuously measured over time. Following the manufacturer's instructions, each channel was calibrated prior to measurement using a gain calibration procedure with electric impulses. Ampoules were first lowered into the thermal equilibration position and left there for $15 \mathrm{~min}$. Then, they were lowered into the measurement position and the heat flow rates recorded for up to 3 days at 10 -second intervals in isothermal conditions at $298 \mathrm{~K}$.

\subsection{Oxygen measurement}

A Fibox 4-trace fiber-optic oxygen meter (PreSens GmbH, Regensburg, Germany) equipped with inner pressure sensors (10-1200 mbar), 5 mm Pst3 luminescence oxygen sensors, resistance 
temperature detectors PT $100\left(0-50^{\circ} \mathrm{C}\right)$ and $2 \mathrm{~mm}$ PMMA fibers was used to measure the oxygen consumption in the same ampoules as used in the calorimeter. The Pst3 oxygen sensors were used for oxygen concentration ranges from 0 to $100 \%$ with LOD $0.03 \%$ and response time $<6$ s. The sensors were glued to the inner surface of the ampoule with silicone (RS components, MörfeldenWalldorf, Germany) at $1 / 2$ height between the bottom and neck before the experiment. Two-point calibration in oxygen-free environment and air-saturated environment was used. Oxygen free water $(100 \mathrm{~mL})$ was obtained by mixing $1 \mathrm{~g}$ sodium sulfite $\left(\mathrm{Na}_{2} \mathrm{SO}_{3}\right)$ and $50 \mu \mathrm{L}$ cobalt nitrate $\left(\mathrm{Co}\left(\mathrm{NO}_{3}\right)_{2}\right)$ standard solution $(1000 \mathrm{mg} / \mathrm{Lin}$ nitric acid $0.5 \mathrm{~mol} / \mathrm{L})$. Air saturated water was obtained by bubbling air, while stirring the solution. To prove the accuracy of the sensors, oxygen measurements were performed in ampoules filled with nitrogen and ambient air prior to measurement. Samples of linseed oil and linseed oil with $0.5 \mathrm{mM}$ of BHT were prepared as previously described. They were loaded in the ampoules of the calorimeter, hermetically sealed, and stored at $298 \mathrm{~K}$ in the dark. The oxygen consumption during storage was measured at regular intervals.

\subsection{Viscosity analysis}

Changes in viscosity during cooling were recorded by using a Discovery HR2 rheometer (TA Instruments, Milan, Italy) mounted with a $40 \mathrm{~mm}$ parallel plate geometry. The experiments were performed for systems containing linseed oil with $5 \%(w / w)$ of apple, orange and sunflower waxes. The temperature was controlled by means of a LT ecocooler 150 (Grant Instruments, Cambridge, UK) connected to the Peltier heater embedded in the lower stainless-steel plate. Molten samples were poured onto the lower plate pre-heated at $353 \mathrm{~K}$. The upper plate was then lowered at the measuring gap of $1 \mathrm{~mm}$. The system was thermally equilibrated for at least 5 min. Temperature ramp was set from 353 to $293 \mathrm{~K}$ at a cooling rate of $6 \mathrm{~K} \cdot \mathrm{min}^{-1}$. Measurements were 
carried out at a shear rate of $2 \mathrm{~s}^{-1}$. The viscosity onset temperature was taken as the onset point obtained intersecting the extrapolated baseline with the extrapolated tangent of the rapid increase of the viscosity.

\subsection{Morphological characterization by polarized light microscopy}

The crystalline structure of samples containing $5 \%(\mathrm{w} / \mathrm{w})$ of apple, orange and sunflower waxes in linseed oil was analyzed using an Axio Lab A1 polarized light microscope (Zeiss, Oberkochen, Germany) connected with an Axiocam 305 color (Zeiss). One drop of the sample was placed in the middle of a glass slide and a glass cover slip was centered above the drop and gently pressed. Samples were analyzed at room temperature using a $20 \times$ objective. Images were acquired and processed using the application software ZEN 2.6 (Zeiss). Images were saved in tiff format resulting in $2464 \times 2056$ pixels.

\subsection{Statistical analysis}

All data were obtained from at least three measurements from two experiment replications and reported as mean value \pm standard deviation. Statistical analysis was performed using Statistica 12.0 software (Statsoft Inc., Hillview Avenue Palo Alto, CA, USA). Bartlett's test was used to check the homogeneity of variance, one-way ANOVA was carried out and Tukey-test was used as posthoc test to determine statistical differences among samples $(\mathrm{p}<0.05)$. Linear regression analysis by least squares minimization was performed using GraphPad Prism v.5.03 (GraphPad Software, San Diego, USA). The goodness of fit was evaluated on the basis of statistical parameters of fitting $\left(\mathrm{R}^{2}\right.$ adj, $\mathrm{p}$-value, standard error) and the residual analysis.

\section{Results and discussion}

\subsection{Linseed oil oxidation by isothermal calorimetry}


Preliminary experiments investigated the oxidation kinetic of linseed oil (certified reference material). Fig 1 shows the raw heat flow trace obtained by isothermal calorimetry from the uninnhibited oxidation of linseed oil at $298 \mathrm{~K}$. Also shown the conversion ratio $(\alpha(t))$, which is given by Eq. 120,21:

$$
\alpha(t)=\frac{q(t)}{Q_{t o t}}
$$

where, $\alpha(t)$ varies from 0 to $1, q(t)$ is the heat obtained by cumulative integration from the heatflow signal $(d q / d t)$ and $Q_{t o t}$ is total area under the heat-flow curve.

The rate of conversion $(d \alpha / d t)$ is negligible at the beginning of the experiment. Suddenly, at about $1.2 \mathrm{~h}$, a sharp break point changes the rate of oxidation (see Fig 1). The onset of such break point identifies two distinct periods: an initial period where the formation of free radicals is initiated (initiation period), followed by a period where linseed oil autoxidation proceeds at high rate (uninhibition period). Consistently, before and after such onset time, two tangent lines can be drawn (see Fig 1, dotted lines). One represents the rate of the initiation period $\left(d \alpha_{i} / d t\right)$, the other is the rate of the uninhibition period $\left(d \alpha_{o x} / d t\right)$.

\subsection{Rate of initiation of linseed oil}

The rate of initiation $\left(R_{i}\right)$ expresses the amount of free reactive radicals generated during a unit of time. A convenient way to determine this rate is by the induction period method $22-24$. Briefly, the method consists of measuring the induction time $(\tau)$, or the length of the inhibition period, when a known amount of a strong antioxidant, like BHT, is used. Then, $R_{i}$ can be determined by Eq. 2:

$$
R_{i}=\frac{n \times[A H]_{0}}{\tau}
$$


where, $[A H]_{0}$ is the initial concentration of BHT; $\tau$ is the induction time and $n$ is the stoichiometry number that is generally assumed to be 2 for $\mathrm{BHT}^{25}$.

Fig 2 shows the conversion ratios of linseed oil autoxidation in the presence of increasing concentrations of BHT. Measurements of $\tau$ give $R_{i}$. Results are reported in Table 2. On average, the rate of initiation $R_{i}$ for linseed oil was equal to $(5.8 \pm 0.1) \times 10^{-8} \mathrm{M} \mathrm{s}^{-1}$. This value is of the same order of that reported in other works when a free radical generator like AIBN and substrates like styrene were used (typically $\left.5.0 \times 10^{-8} \mathrm{M} \mathrm{s}^{-1}\right)^{26}$. However, here radicals are quickly formed from the oxidation of polyunsaturated fatty acid esters, which in linseed oil constitute about $90 \%$, partitioned into linolenic (47\%), linoleic (24\%) and oleic (19\%) acid. Since linseed oil alone seems providing a sufficient and constant rate of radicals formation, no azo-initiators were used in the following experiments. 
Fig 3 reports the changes in the amount of oxygen in the ampoule. When the ampoule was filled with linseed oil, the partial pressure of oxygen remained nearly constant during the earlier initiation period. Afterwards, the partial pressure dropped, consistently with the rapid consumption of oxygen by the large excess of lipid substrate. When BHT was mixed with the lipid substrate, the partial pressure of oxygen remained nearly constant as far as the antioxidant was present. Only when the activity of the antioxidant was over, then, the partial pressure of oxygen dropped similarly to the previous case, i.e. when only the lipid substrate was present. These evidences confirm that the initial amount of oxygen in the ampoule was enough to induce the oxidation of the lipid substrate. Also, thanks to the large amount of substrate used, the duration of the inhibited phase, i.e. when antioxidants inhibit lipid oxidation, is emphasized.

\subsection{Effect of orange and apple waxes on linseed oil autoxidation}

The effect of orange and apple waxes on the autoxidation of linseed oil was investigated by studying the inhibited period. In details, the rate of inhibited oxidation $\left(d \alpha_{i} / d t\right)$ was used to represent the specific activity of extracted waxes, whereas the length of the inhibited period $\tau$ was used to express the product between stoichiometry and concentration of the actives present in the waxes $^{27}$. Fig 4 shows the conversion ratios of linseed oil when mixed with apples and orange peel waxes. For comparison, the conversion ratios of linseed oil without antioxidants (control sample) and fortified with BHT are also shown as dotted lines. The activity of the waxes falls in between these two extremes, with apple waxes more active than orange waxes. However, a few differences should be highlighted. First, although waxes can slow linseed oxidation, however, such activity is visible only at much higher concentrations ( $>1 \%$ of waxes) than the ones used by radical chain breakers, like BHT. Also, the use of waxes led to a flatter elbow curve between the inhibited and uninhibited periods, which resulted in a less definite induction period. Table 3 reports such 
induction periods obtained with increasing concentrations of waxes. The resulting dose/response curves confirmed a monotonic correlation only for apple waxes $\left(\mathrm{R}^{2}=0.99\right.$, slope $=0.32 \pm 0.1$. $\left.10^{4} \mathrm{~s} \%{ }^{-1}\right)$. However, a dose/response relationship is absent for orange waxes.

\subsection{Mechanism of inhibition of linseed oil oxidation by orange and apple waxes}

The behavior of waxes to inhibit linseed oil oxidation is markedly different from the classical chain-breaking activity of BHT. To explain such behavior, three possible mechanisms have been considered. The first accounts for a residual presence of natural antioxidants in waxes. This was verified here by measuring the residual polyphenol content in waxes. Whitaker et al. have reported that phenolic fatty acid esters are present in the epicuticular wax apple fruit as $\mathrm{E}$ and $\mathrm{Z}$ isomers of p-coumaryl alcohol with primarily long-chain, namely the E-p-coumaryl alcohol and the Z-pcoumaryl alcohol. In addition, the $\gamma$-O-methyl and O-ethyl ethers of E-p-coumaryl alcohol are also released from the apple phenolic esters among as free phenolics with antioxidant activity ${ }^{28}$. Similarly, in orange waxes the radical scavenging ability is associated to the presence of methyl esters of fatty acids such as tetradecanoic acid, 12-methyl ester, or 1,2,3,4,5-cyclopentanepentol ${ }^{29}$.

In this study, results showed that apples waxes have a higher content of polyphenols $(1.7 \pm 0.1 \mathrm{mg}$ $\mathrm{GAE} \cdot \mathrm{g}^{-1}$ wax $)$ than orange waxes $\left(0.7 \pm 0.2 \mathrm{mg} \mathrm{GAE} \cdot \mathrm{g}^{-1}\right.$ wax $)$. The fact that apple waxes have twice phenols concentration than orange waxes is consistent with their longer induction time. However, it should be stressed that the inhibitory behavior observed with waxes is not consistent with a classical chain-breaking activity. The need of high waxes concentrations respect to the sample and the lack of linear correlation between the dose of orange waxes and the resulting induction time is not consistent with a classical chain breaking activity. Overall, the presence of phenols in the waxes cannot be the only reason to explain the inhibition activity of the waxes. 
A second intriguing mechanism was recently proposed by Baschieri et al. to explain the complex antioxidant behavior of non-phenolic essential oils ${ }^{30}$. In that work, it was hypothesized that essential oils can initiate themselves the radical chain reaction by generating peroxyl radicals that can undergo quick self-termination reaction. In other words, faster termination reactions lower the concentration of peroxyl radicals in the mixture and, consequently, slow down the overall rate of oxidation $^{31}$. If such hypothesis can be applied to waxes, we tested the capacity of purified sunflower wax (free from phenols, as determined by Folin-Ciocalteu method) to inhibit linseed oil oxidation. Fig 5 shows the resulting conversion ratio from increasing concentrations of sunflower wax.

Notably, sunflower waxes showed a superior capacity to delay the rate of linseed oil oxidation, showing longer induction times than apple and orange waxes. Surprisingly, the capacity of sunflower wax to retard linseed oil oxidation in terms of induction period is proportional to the wax concentration $\left(\mathrm{R}^{2}=0.99\right.$, slope $\left.=2.1 \pm 0.310^{4} \mathrm{~s} \%{ }^{-1}\right)$, although only at very high doses $(>$ 1\%). By the way, Fig 5 also shows the conversion factor observed when no linseed oil was present (dashed line). In such case, the wax sample did not exhibit any measurable exothermic signal. Form one side, these results confirm that waxes have some inhibition activity towards linseed oil oxidation. From the other, waxes themselves cannot generate free radicals in an amount comparable with linseed oil. Thus, we can conclude that the inhibition activity exerted by waxes cannot be explained (entirely) by their chain termination-enhancing capacity.

The third mechanism that could tentatively explain the enhanced inhibition activity of waxes on linseed oil oxidation is based on a physical hindrance. In details, during the preparation of the linseed oil/wax mixture, the temperature was held at $353 \mathrm{~K}$ for 20 minutes in order to properly mix the two solutions. Afterwards, during the cooling step, a change in the viscosity of the lipid/wax 
system was observed. Fig 6 shows the changes in viscosity observed during the cooling of mixture of linseed oil with orange, apple and sunflower wax $\left(6 \mathrm{~K} \mathrm{~min}^{-1}\right)$. What is striking here is that all samples exhibit a great change in their viscosity during their cooling to $293 \mathrm{~K}$. In particular, the mixture containing sunflower, apple and orange waxes changed their viscosity from about 0.010 $0.020 \mathrm{~Pa} \cdot \mathrm{s}$ at $338 \mathrm{~K}$ to, respectively, $104 \mathrm{~Pa} \cdot \mathrm{s}, 0.321 \mathrm{~Pa} \cdot \mathrm{s}$ and $0.0437 \mathrm{~Pa} \cdot \mathrm{s}$ at $293 \mathrm{~K}$.

The results indicate that different type of waxes have different capacity to form crystal networks with linseed oil during cooling. The crystallization process starts at higher temperatures for sunflower waxes and lower for apples and orange waxes ${ }^{32-34}$.

Moreover, to test the presence of a physical hindrance behind the enhanced oxidative stability of linseed oil mixed with waxes, different oil/wax system have been prepared by cooling the mixture at different rates. Fig 7 shows the effect cooling rate on the linseed oil oxidation prepared with sunflower wax. Cooling rates have a strong influence on the resulting oxidation rate of linseed oil, confirming the importance of the physical hindrance on the oxidation. In details, at lower cooling rates, the lipid/wax system showed also the lowest viscosity. However, by increasing the cooling rate up to $6 \mathrm{~K} \mathrm{~min}^{-1}$, the viscosity also increased, indicating the best condition for producing crystal networks. Changes in viscosity directly affected the rate of diffusion of oxygen. From the Stokes Einstein relationship, the diffusion of oxygen in the lipid substrates can be calculated as $1.54 \cdot 10^{-}$ ${ }^{10}, 6.20 \cdot 10^{-8}$, and $4.54 \cdot 10^{-7} \mathrm{~cm}^{2} \cdot \mathrm{s}^{-1}$ (with the hydrodynamic radius of oxygen equal to $110 \mathrm{pm}$ and temperature of $298 \mathrm{~K}$ ), respectively for sunflower, apple and oranges waxes. A further increase of the cooling rate did not seem to enhance more the physical inhibition exerted by waxes.

Faster cooling rates did not seem to enhance further the physical inhibition exerted by the waxes. The crystal network formed by waxes was confirmed by polarized light microscopy. Fig 8 shows the micrographs of systems containing sunflower and apple peel waxes obtained at $6 \mathrm{~K} \mathrm{~min}^{-1}$. 
Bright areas refer to crystalline waxes, while dark areas represent liquid oil. Sunflower waxes showed a network of interconnected platelet-like crystals with dimension of approximately 10-20 $\mu \mathrm{m}$ (Fig 8 A). Instead, Fig 8 B shows the microstructure of a system containing apple waxes. The results show that systems based on apple (or orange) waxes have a minor capacity to form crystalline structures. Overall, the ability of waxes to form a more compact crystal network, which resulted in a structured system, can be likely responsible to act as a barrier toward oxygen diffusion and, thus, slowing down the oxidation process of linseed oil.

\subsection{Significance of vegetable waxes for the protection of food lipids}

Despite waxes cannot exert a classical chain breaking activity, their capacity to inhibit or retard lipid oxidation can be still of significance for food applications. For instance, waxes can sustain or amplify the radical scavenging activity of primary antioxidants. Such possibility was demonstrated in Fig 9. In this experiment, mixtures of wax, primary antioxidants and linseed oil were mixed together, heated the mixture at $353 \mathrm{~K}$ for $20 \mathrm{~min}$ and, then, cooled down to $298 \mathrm{~K}$ at a constant rate of $6 \mathrm{~K} \mathrm{~min}^{-1}$. The resulting mixtures exhibited longer induction periods than the sum of the induction periods obtained from the individual use of BHA, ethoxyquin, $\alpha$-tocopherol or waxes (after all have undergone the same treatment). This cooperative enhancement of the oxidative stability observed with the combination of classical chain breaking antioxidants and vegetable waxes pave the way to the development of novel antioxidant systems.

\section{Conclusions}

In this study a study on the antioxidant activity of vegetable waxes extracted by supercritical carbon dioxide has been performed by isothermal calorimetry. The antioxidant activity of apples and orange waxes was evaluated by the induction period method from the kinetic of linseed oil oxidation performed under aerobic conditions without the need of initiators. The results indicated 
that the waxes were able to inhibit linseed oil autoxidation reaction with apple waxes more active than orange waxes. However, such activity was obtained only at much higher concentrations (> $1 \%$ of waxes) than the ones used by a synthetic antioxidant like BHT added to linseed oil. The comparison with a commercial sunflower wax demonstrated its superior capacity to delay the rate of linseed oil oxidation as indicated by the longer induction times than those of apple and orange waxes. The enhanced inhibition activity of sunflower wax on linseed oil oxidation was explained by considering its ability to form crystal networks within the oil during cooling. As concerns the combination of waxes with primary antioxidants, the results showed that the systems composed by the mixture of linseed oil, sunflower wax and BHA, ethoxyquin or $\alpha$-tocopherol exhibited an induction period longer than the sum of the induction periods obtained from the individual use of BHA, ethoxyquin, $\alpha$-tocopherol or wax. This finding clearly showed the potential of vegetable waxes enhancing their application as antioxidant agents thanks to their ability of amplifying the radical scavenging activity of primary antioxidants.

\section{Conflict of interest}

There are no conflicts to declare.

\section{Acknowledgement}

We are grateful to the Province of Bolzano for financial support (Landesregierung mittels Beschluss Nr. 1472, 07.10.2013).

\section{References}

1. S. Martini and M. Añón. Crystallization of sunflower oil waxes, J Am Oil Chem Soc, 2003, 80, 525-532. 
2. S. R. Vali, Y. Ju, T.N.B. Kaimal and Y. Chern, A process for the preparation of food-grade rice bran wax and the determination of its composition, J Am Oil Chem Soc, 2005, 82, 57-64.

3. G. F. Mehyar, K. Al-Ismail, J.H. Han and G.W. Chee, Characterization of edible coatings consisting of pea starch, whey protein isolate, and carnauba wax and their effects on oil rancidity and sensory properties of walnuts and pine nuts, J Food Sci, 2012, 77, E52-E59.

4. L.B. da Silva Andrade, J. da Silva, S. Murilo, R.C.V. Cruz, T.H.S Rodrigues, F. dos Santos, R. Oliveira and A.L.C da Silva, Antioxidant and antifungal activity of carnauba wax powder extracts, Industrial Crops and Products, 2018, 125, 220-227.

5. T. Osawa and M. Namiki, A novel type of antioxidant isolated from leaf wax of Eucalyptus leaves, Agric Biol Chem, 1981, 45, 735-739.

6. S. Mezouari, S.P. Kochhar, K. Schwarz and K. Eichner, Effect of dewaxing pretreatment on composition and stability of rice bran oil: Potential antioxidant activity of wax fraction, Eur $J$ Lipid Sci Tech, 2006, 108, 679-686.

7. E. Yılmaz and M. Öğütcü, Properties and stability of hazelnut oil organogels with beeswax and monoglyceride, J Am Oil Chem Soc, 2014, 91, 1007-1017.

8. M.A. Rogers, A.J. Wright and A.G. Marangoni, Oil organogels: the fat of the future?, Soft Matter, 2009, 5, 1594-1596.

9. A.R. Patel and K. Dewettinck, Edible oil structuring: an overview and recent updates, Food \& function, 2016, 7, 20-29. 
10. D.C. Edmund and A.G. Marangoni, Organogels: an alternative edible oil-structuring method, J Am Oil Chem Soc, 2012, 89, 749-780.

11. M. Öğütcü, N. Arifoğlu and E. Yılmaz, Storage stability of cod liver oil organogels formed with beeswax and carnauba wax, Int J Food Sci Tech, 2015, 50, 404-412.

12. B. Yi, M. Kim, S.Y. Lee and J. Lee, Physicochemical properties and oxidative stability of oleogels made of carnauba wax with canola oil or beeswax with grapeseed oil, Food science and biotechnology, 2017, 26, 79-87.

13. H. Hwang, M. Fhaner, J.K. Winkler-Moser and S.X. Liu, Oxidation of fish oil oleogels formed by natural waxes in comparison with bulk oil, Eur J Lipid Sci Tech, 2018, 120, 1700378.

14. N. Martinović, U.N. Poklar and H. Abramovič, Sinapic acid and its derivatives increase oxidative stability in different model lipid systems, Eur J Lipid Sci Tech, 2019, 121, 1800326.

15. M. Öğütcü, R. Temizkan, N. Arifoğlu and E. Y1lmaz, Structure and stability of fish oil organogels prepared with sunflower wax and monoglyceride, Journal of oleo science, 2015, 64, 713-720.

16. N. Haman, A. Romano, M. Asaduzzaman, G. Ferrentino, F. Biasioli and M. Scampicchio, A microcalorimetry study on the oxidation of linoleic acid and the control of rancidity, Talanta, 2017 , $164,407-412$.

17. N. Haman, M. Bodner, G. Ferrentino and M. Scampicchio, Lipid autoxidation of fish, lard, corn and linseed oils by isothermal calorimetry, Italian Journal of Food Science, 2019, 31, 323331. 
18. N. Haman, G. Ferrentino, S. Imperiale and M. Scampicchio, Antioxidant and prooxidant activity of spent coffee extracts by isothermal calorimetry, Journal of Thermal Analysis and Calorimetry, 2018, 132, 1065-1075.

19. V. Singleton and J.A. Rossi, Colorimetry of total phenolics with phosphomolybdicphosphotungstic acid reagents, American Journal of Enology and Viticulture, 1965, 16, 144-158.

20. G. Litwinienko, T. Kasprzycka-Guttman and M. Jarosz-Jarszewska, Dynamic and isothermal DSC investigation of the kinetics of thermooxidative decomposition of some edible oils, Journal of Thermal Analysis, 1995, 45, 741-750.

21. S. Fujisawa and Y. Kadoma, Comparative study of the alkyl and peroxy radical scavenging activities of polyphenols, Chemosphere, 2006, 62, 71-79.

22 S. Fujisawa, M. Ishihara and Y. Kadoma, Kinetic evaluation of the reactivity of flavonoids as radical scavengers, SAR QSAR Environ. Res., 2002, 13, 617-627.

23. C.E. Boozer, G.S. Hammond, C.E. Hamilton and J.N. Sen, Air oxidation hydrocarbons. II. The stoichiometry and fate of inhibitors in benzene and chlorobenzene, J. Am. Chem. Soc., 1995, 77, $3233-3237$.

24. M. Lucarini and G.F. Pedulli, Free radical intermediates in the inhibition of the autoxidation reaction, Chemical Society Reviews, 2010, 39, 2106-2119.

25. G.W. Burton and K.U. Ingold, Autoxidation of Biological Molecules. 1. The Antioxidant Activity of Vitamin E and Related Chain-Breaking Phenolic Antioxidants in Vitro, J. Am. Chem. Soc., 1981, 103, 6472-6477. 
26. L. Valgimigli, G. Brigati, G.F. Pedulli, G.A. Di Labio, M. Mastragostino, C. Arbizzani and D.A. Pratt, The effect of ring nitrogen atoms on the homolytic reactivity of phenolic compounds: understanding the radical-scavenging ability of 5-pyrimidinols, Chem. Eur. J., 2003, 9, 4997 5010.

27. R. Amorati and L. Valgimigli, Methods to measure the antioxidant activity of phytochemicals and plant extracts, J. Agric. Food Chem., 2018, 66, 3324-3329.

28. B.D. Whitaker, W.F. Schmidt, M.C. Kirk and S. Barnes, Novel Fatty Acid Esters of pCoumaryl Alcohol in Epicuticular Wax of Apple Fruit, J Agric Food Chem, 2001, 49, 3787-3792.

29. O.L. Erukainure, O.A.T. Ebuehi, M.I. Chaudhary, M. A. Mesaik, A. Shukralla, A. Muhammad, M.Z. Zaruwa and G.N. Elemo, Orange Peel Extracts: Chemical Characterization, Antioxidant, Antioxidative Burst, and Phytotoxic Activities, Journal of Dietary Supplements, 2016, 1-10.

30. A. Baschieri, M.D. Ajvazi, J.L.F. Tonfack, L. Valgimigli and R. Amorati, Explaining the antioxidant activity of some common non-phenolic components of essential oils, Food Chemistry, $2017,232,656-663$.

31. R. Amorati and G.F. Pedulli, Do garlic-derived allyl sulfides scavenge peroxyl radicals?, Org. Biomol. Chem., 2008, 6, 1103-1107.

32. G. Ferrentino, K. Morozova, O.K. Mosibo, M. Ramezani and M. Scampicchio, Biorecovery of antioxidants from apple pomace by supercritical fluid extraction, J Clean Prod, 2018, 186, 253261. 
33. J. Li, Y. Guo, Z. Li, Y. Lin, L. Liu and X. Zhang, Supercritical carbon dioxide and hexane extraction of wax from apple peel pomace: Content, composition, and thermal properties, Sep Sci Technol, 2015, 50, 2230-2237.

34. A.I. Blake, E.D. Co and A.G. Marangoni, Structure and physical properties of plant wax crystal networks and their relationship to oil binding capacity, J Am Oil Chem Soc, 2014, 91, 885-903.

Table 1 Chemical characterization of apple and orange waxes obtained by supercritical fluid extraction.

\begin{tabular}{|c|c|c|c|c|}
\hline $\begin{array}{c}\text { Type of } \\
\text { compound }\end{array}$ & Compound & $\begin{array}{l}\text { Retention time } \\
\text { (min) }\end{array}$ & $\begin{array}{c}\text { Orange } \\
(\%)\end{array}$ & $\begin{array}{c}\text { Apple } \\
(\%)\end{array}$ \\
\hline \multirow[t]{8}{*}{ Terpenoids } & B-sitosterol & 24.603 & $0.62 \pm 0.03$ & $1.82 \pm 0.09$ \\
\hline & $\alpha$-amyrin & 24.801 & $7.51 \pm 0.38$ & n.d. \\
\hline & ß-amyrin & 25.025 & $8.46 \pm 0.42$ & n.d. \\
\hline & Lupeol acetate & 25.692 & $10.23 \pm 0.52$ & n.d. \\
\hline & Friedlin & 26.234 & $14.11 \pm 0.71$ & n.d. \\
\hline & Squalene & 26.876 & $16.18 \pm 0.81$ & n.d. \\
\hline & Farnesol & 27.012 & $4.57 \pm 0.23$ & n.d. \\
\hline & Agnosterol & 27.409 & n.d. & $0.67 \pm 0.03$ \\
\hline \multirow[t]{8}{*}{ Fatty acids } & $\mathrm{C} 14$ & 8.627 & $1.26 \pm 0.06$ & n.d. \\
\hline & $\mathrm{C} 16$ & 9.972 & $1.48 \pm 0.07$ & $2.98 \pm 0.15$ \\
\hline & $\mathrm{C} 18$ & 11.683 & n.d. & $1.67 \pm 0.08$ \\
\hline & $\mathrm{C}(18: 1)$ & 11.474 & n.d. & $3.64 \pm 0.11$ \\
\hline & $C(18: 2)$ & 11.442 & n.d. & $4.63 \pm 0.19$ \\
\hline & $\mathrm{C} 24$ & 18.532 & $2.67 \pm 0.13$ & n.d. \\
\hline & $\mathrm{C} 26$ & 25.792 & n.d. & $1.95 \pm 0.10$ \\
\hline & $\mathrm{C} 30$ & 24.426 & $3.89 \pm 0.19$ & n.d. \\
\hline \multirow{7}{*}{$\begin{array}{l}\text { Primary } \\
\text { alcohols }\end{array}$} & $\mathrm{C} 21$ & 14.491 & n.d. & $20.47 \pm 1.02$ \\
\hline & $\mathrm{C} 24$ & 17.341 & n.d. & $2.52 \pm 0.10$ \\
\hline & $\mathrm{C} 25$ & 18.542 & $1.56 \pm 0.08$ & n.d. \\
\hline & $\mathrm{C} 26$ & 19.809 & n.d. & $7.43 \pm 0.22$ \\
\hline & $\mathrm{C} 28$ & 22.164 & n.d. & $7.22 \pm 0.14$ \\
\hline & $\mathrm{C} 30$ & 24.426 & n.d. & $1.25 \pm 0.05$ \\
\hline & 1,30-Triacontane diol & 23.660 & n.d. & $2.48 \pm 0.12$ \\
\hline \multirow[t]{4}{*}{ Alcanes } & $\mathrm{C} 25$ & 14.472 & $1.27 \pm 0.06$ & $0.51 \pm 0.02$ \\
\hline & $\mathrm{C} 27$ & 16.878 & $1.96 \pm 0.10$ & $1.41 \pm 0.03$ \\
\hline & $\mathrm{C} 29$ & 19.480 & $4.35 \pm 0.22$ & $34.24 \pm 1.71$ \\
\hline & $\mathrm{C} 31$ & 21.840 & $3.83 \pm 0.19$ & n.d. \\
\hline \multirow[t]{3}{*}{ Aldehydes } & $\mathrm{C} 24$ & 16.248 & $3.15 \pm 0.16$ & n.d. \\
\hline & $\mathrm{C} 28$ & 18.342 & $4.25 \pm 0.21$ & n.d. \\
\hline & $\mathrm{C} 30$ & 20.432 & $2.51 \pm 0.12$ & n.d. \\
\hline
\end{tabular}


Table 2 Rate of initiation and induction time of linseed oil with increasing concentration of BHT obtained by calorimetric traces at $298 \mathrm{~K}$ in aerobic conditions.

\begin{tabular}{ccc}
\hline BHT & $\boldsymbol{\tau}$ & $\mathbf{R}_{\mathbf{i}}$ \\
$\mathrm{M} \mathrm{x} 10^{3}$ & $\mathrm{~s} \mathrm{x} 10^{4}$ & $\mathrm{M} \mathrm{s}^{-1} \times 10^{-8}$ \\
\hline 0.0 & $1.5 \pm 0.2$ & - \\
0.5 & $2.6 \pm 0.1$ & $5.8 \pm 0.1$ \\
1.0 & $5.0 \pm 0.5$ & $5.6 \pm 0.3$ \\
2.0 & $8.2 \pm 0.3$ & $5.9 \pm 0.2$ \\
2.5 & $10.0 \pm 0.4$ & $5.8 \pm 0.2$ \\
\hline
\end{tabular}


Table 3 Duration of inhibition phase $(\tau)$ and rate of the inhibited oxidation $\left(d \alpha_{i} / d t\right)$ of linseed oil mixed with increasing concentrations of apple and orange waxes.

\begin{tabular}{|c|c|c|c|}
\hline Wax & $\begin{array}{c}\text { Concentration } \\
\%\end{array}$ & $\begin{array}{c}\boldsymbol{d} \boldsymbol{\alpha}_{\boldsymbol{i}} / \boldsymbol{d} \boldsymbol{t} \\
\mathrm{s}^{-1} \times 10^{-6}\end{array}$ & $\begin{array}{c}\tau \\
\mathrm{s} \times 10^{4}\end{array}$ \\
\hline- & 0.0 & - & $1.5 \pm 0.2$ \\
\hline Apple & $\begin{array}{l}0.5 \\
1.0 \\
2.0 \\
3.0 \\
5.0\end{array}$ & $\begin{array}{l}0.91 \pm 0.11 \\
0.85 \pm 0.12 \\
0.73 \pm 0.12 \\
0.68 \pm 0.15 \\
0.46 \pm 0.13\end{array}$ & $\begin{array}{l}1.71 \pm 0.14 \\
1.82 \pm 0.21 \\
2.14 \pm 0.21 \\
2.53 \pm 0.22 \\
3.12 \pm 0.31\end{array}$ \\
\hline Orange & $\begin{array}{l}0.5 \\
1.0 \\
2.0 \\
3.0 \\
5.0\end{array}$ & $\begin{array}{l}2.44 \pm 0.11 \\
2.71 \pm 0.10 \\
2.54 \pm 0.13 \\
2.51 \pm 0.11 \\
2.46 \pm 0.12\end{array}$ & $\begin{array}{l}1.51 \pm 0.12 \\
1.63 \pm 0.22 \\
1.91 \pm 0.32 \\
2.11 \pm 0.43 \\
2.62 \pm 0.52\end{array}$ \\
\hline
\end{tabular}




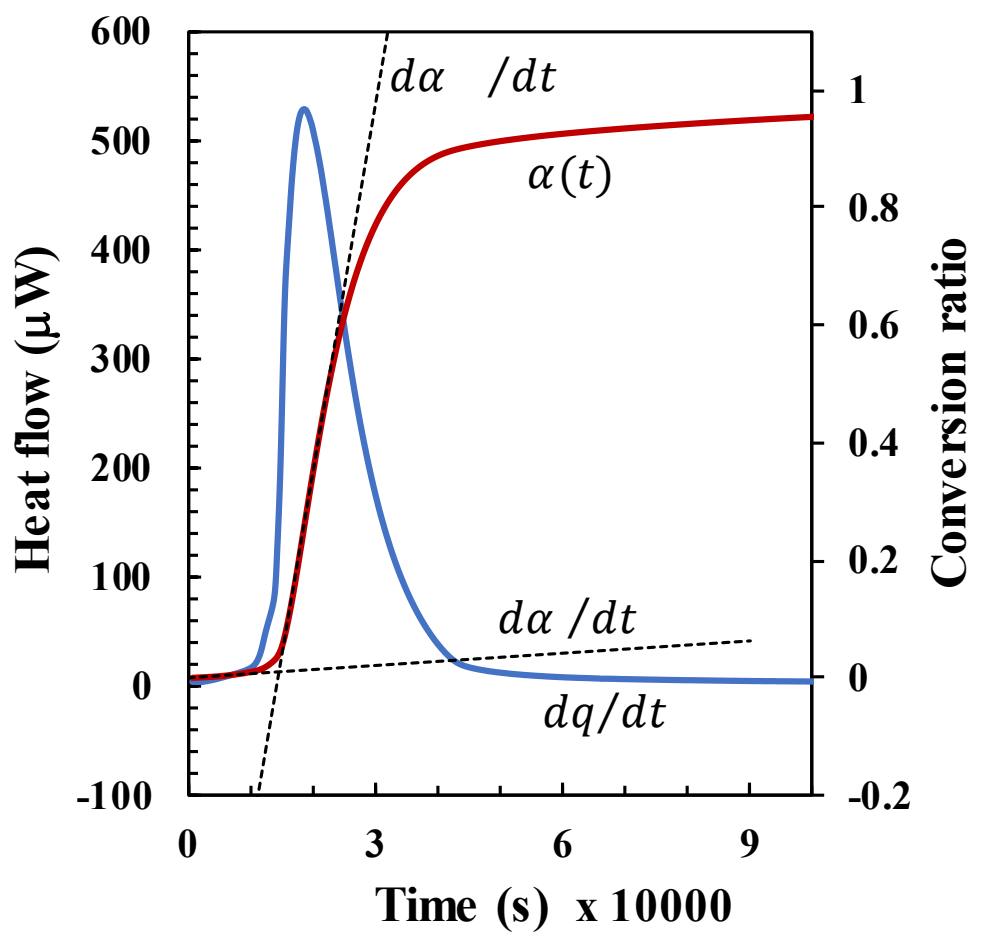

Fig 1 Calorimetric trace of linseed oil measured as heat-flow (blue curve) and ratio of conversion (red curve) at $298 \mathrm{~K}$ in aerobic conditions. 


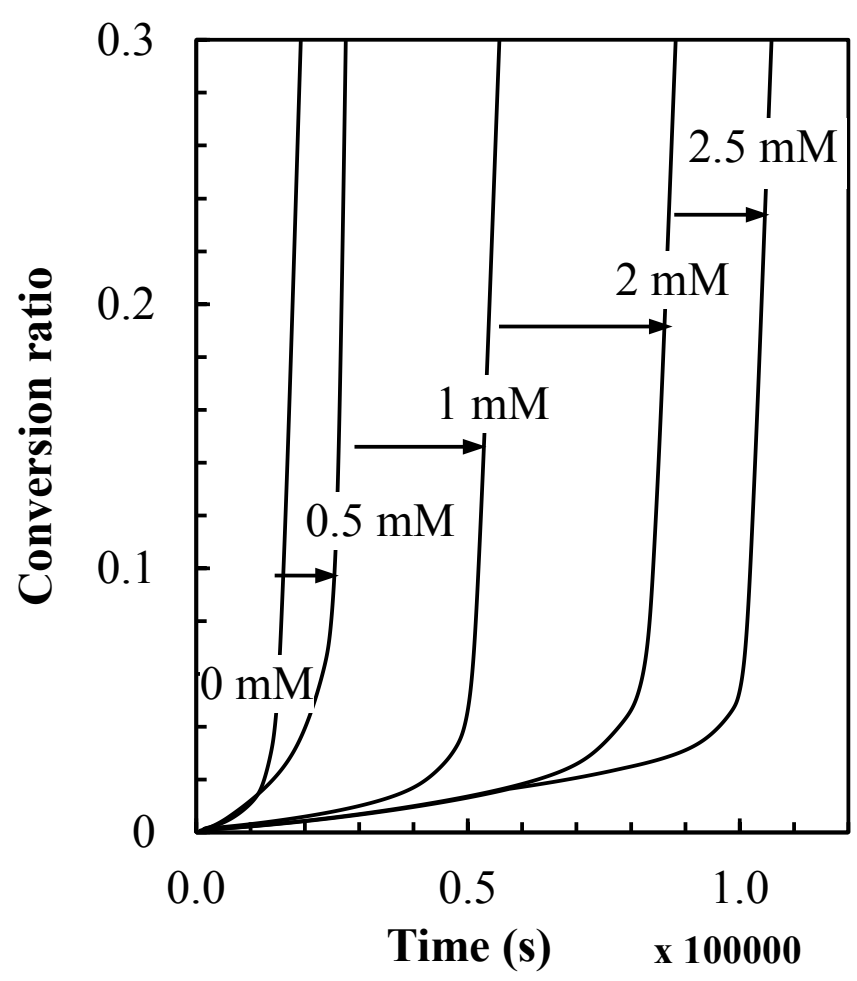

Fig 2 Conversion ratios for the autoxidation of linseed oil in the presence of increasing concentrations of BHT $(0,0.5,1,2$ and $2.5 \mathrm{mM})$ at $298 \mathrm{~K}$ in aerobic conditions. 


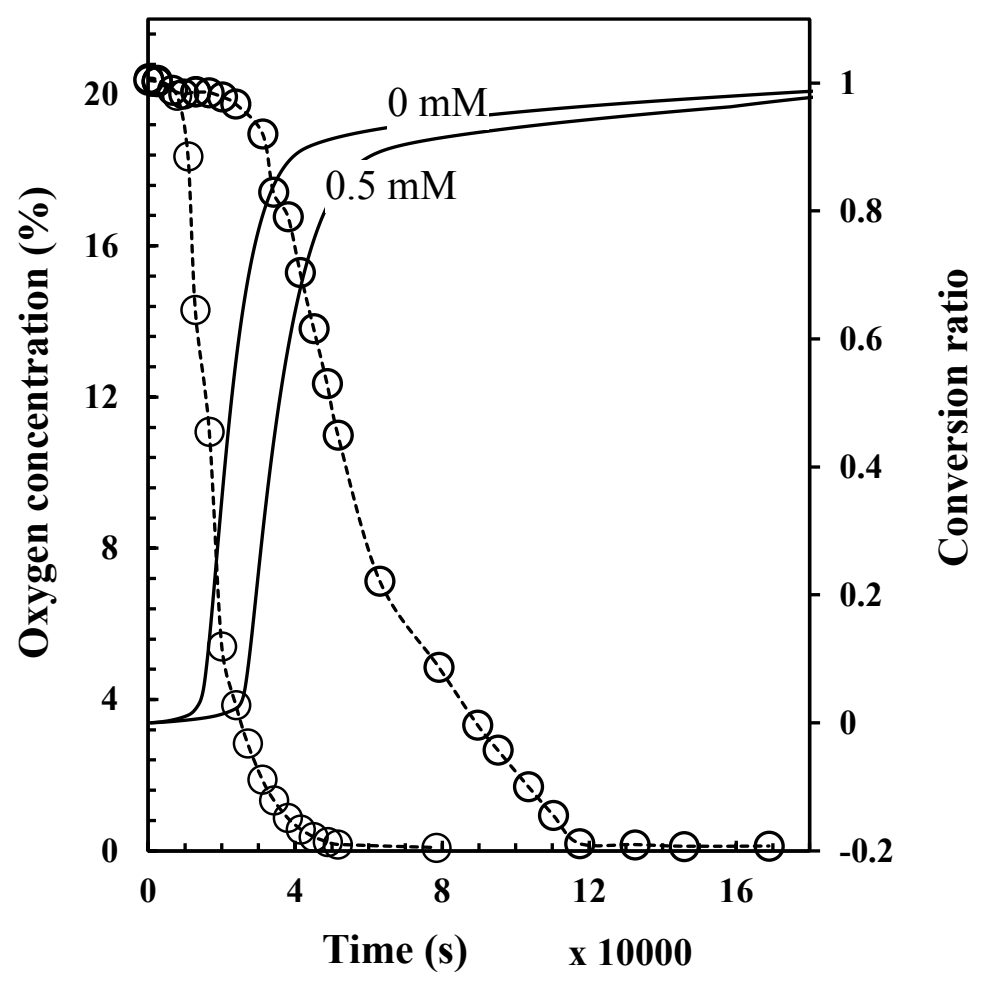

Fig 3 Oxygen concentration and conversion ratio for the autoxidation of linseed oil $(0 \mathrm{mM})$ and linseed oil with BHT $(0.5 \mathrm{mM})$ at $298 \mathrm{~K}$ in aerobic conditions. 


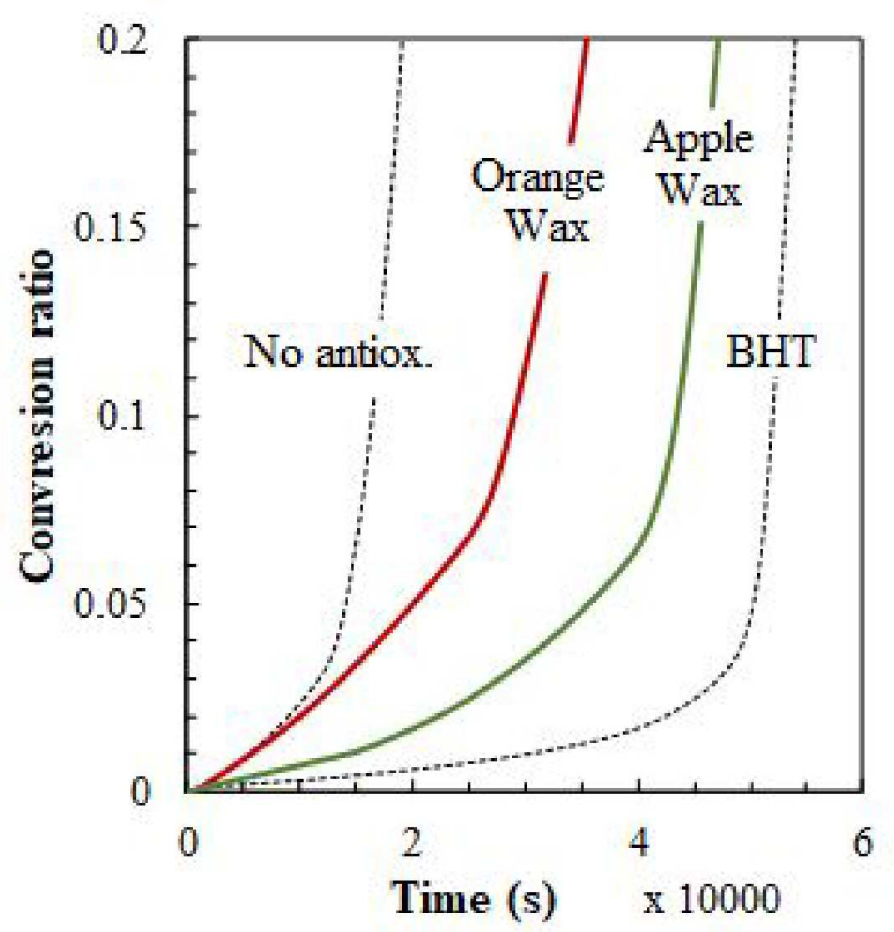

Fig 4 Conversion degree curves obtained by isothermal calorimetry of orange and apple peel waxes extracted by supercritical carbon dioxide for the samples: untreated linseed oil (no antiox.); linseed oil mixed with BHT (1 mM); linseed oil mixed with orange peel waxes at concentrations of $2 \%$; linseed oil mixed with apple peel waxes at concentrations of $2 \%$. These samples were prepared by mixing the oil with waxes at $353 \mathrm{~K}$ for $20 \mathrm{~min}$, following cooling at controlled rate of $6.0 \mathrm{~K} \mathrm{~min}^{-1}$. 


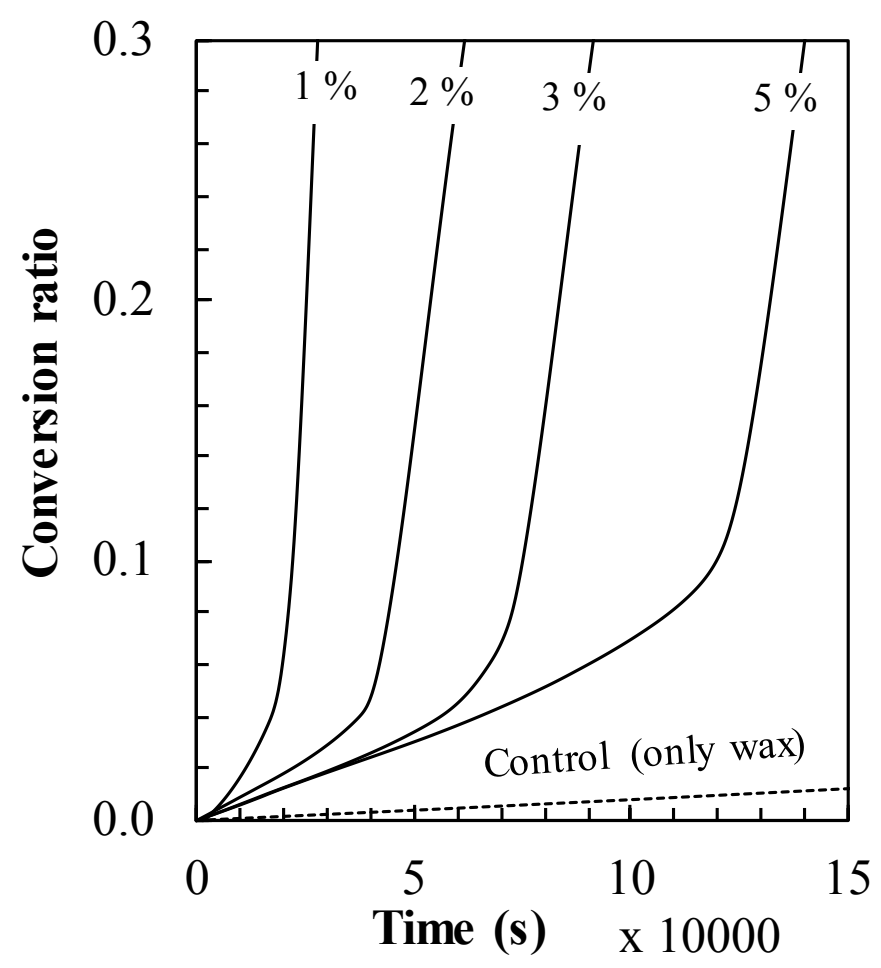

Fig 5 Conversion degree curves obtained by isothermal calorimetry of sunflower wax added to linseed oil at increasing concentrations $(1,2,3$ and $5 \% \mathrm{w} / \mathrm{w})$. The samples were prepared by mixing the oil with waxes at $353 \mathrm{~K}$ for $20 \mathrm{~min}$, following cooling at controlled rate of $6.0 \mathrm{~K} \mathrm{~min}^{-}$ ${ }^{1}$. The conversion ratio of the dotted line is the control sample containing only wax (without linseed oil). This was determined by dividing the cumulative heat by the average $\mathrm{Q}_{\text {tot }}$. 


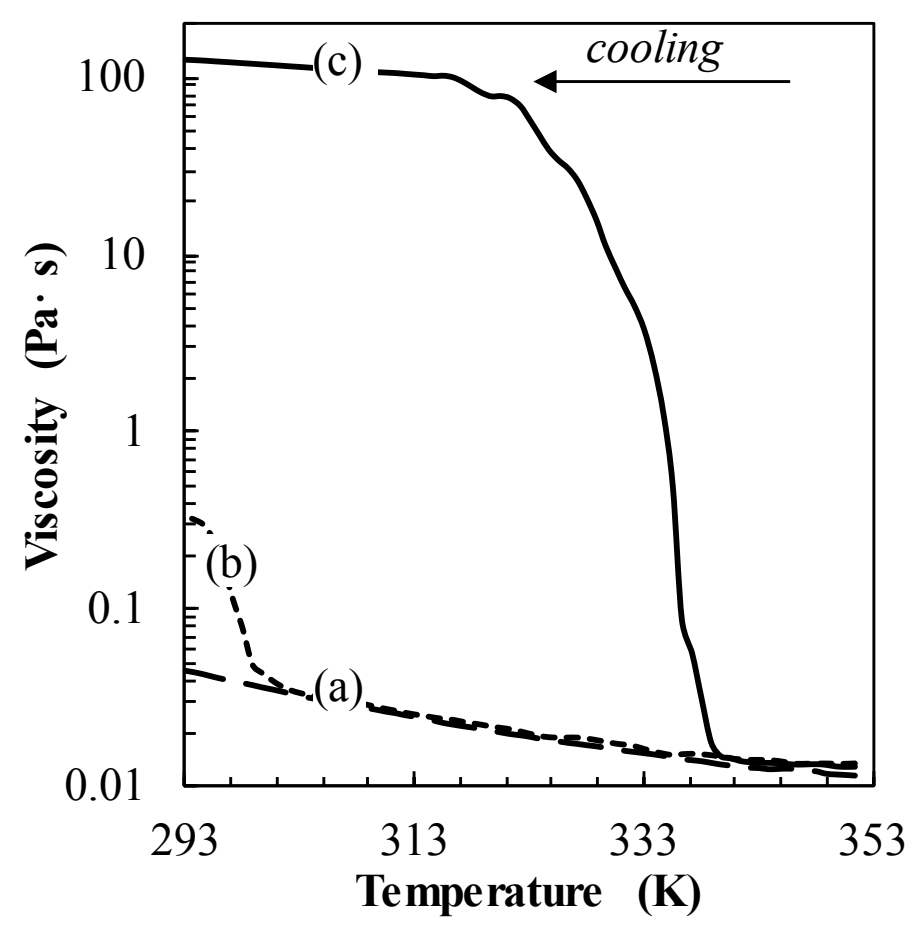

Fig 6 Viscosity changes during the cooling of the samples for concentrations of orange (a), apple (b) and sunflower wax (c) equal to $5 \%(\mathrm{w} / \mathrm{w})$. For all traces, cooling rate was $6.0 \mathrm{~K} \cdot \mathrm{min}^{-1}$ 


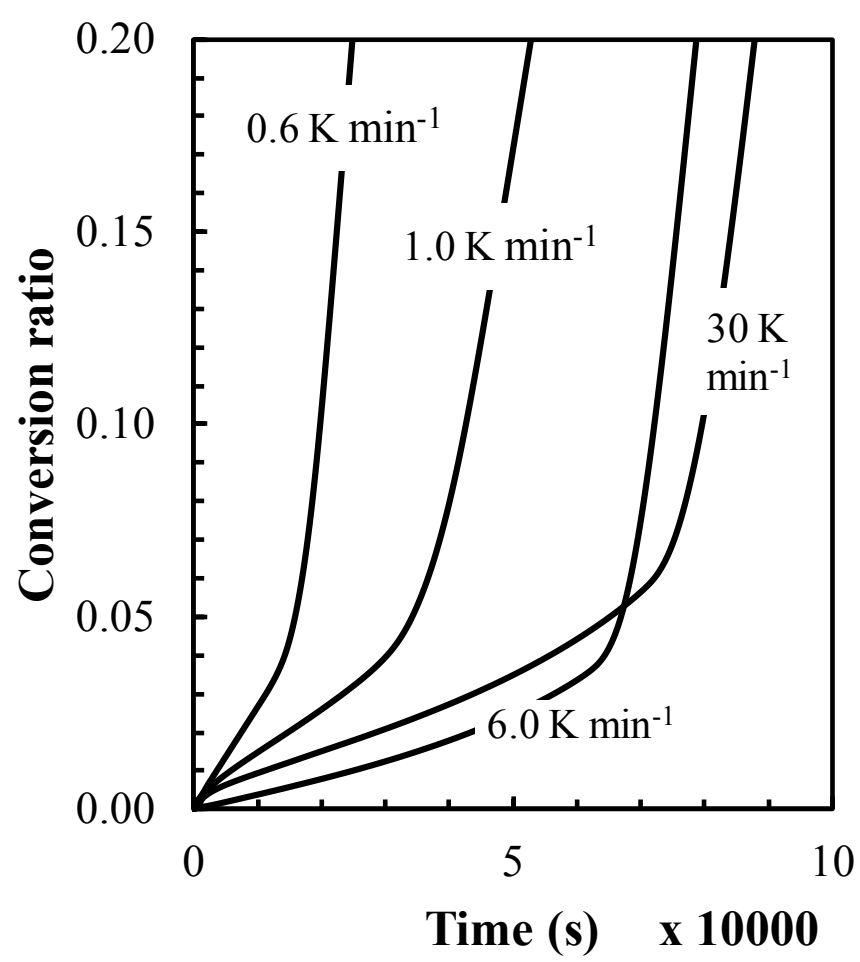

Fig 7 Conversion degree curves obtained by isothermal calorimetry of sunflower wax added to linseed oil at concentration $(3 \%, \mathrm{w} / \mathrm{w})$. The samples were prepared by mixing the oil with wax at $353 \mathrm{~K}$ for $20 \mathrm{~min}$, following cooling at different controlled rate of $0.6,1.0,6.0$ and $30 \mathrm{~K} \mathrm{~min}^{-1}$. 

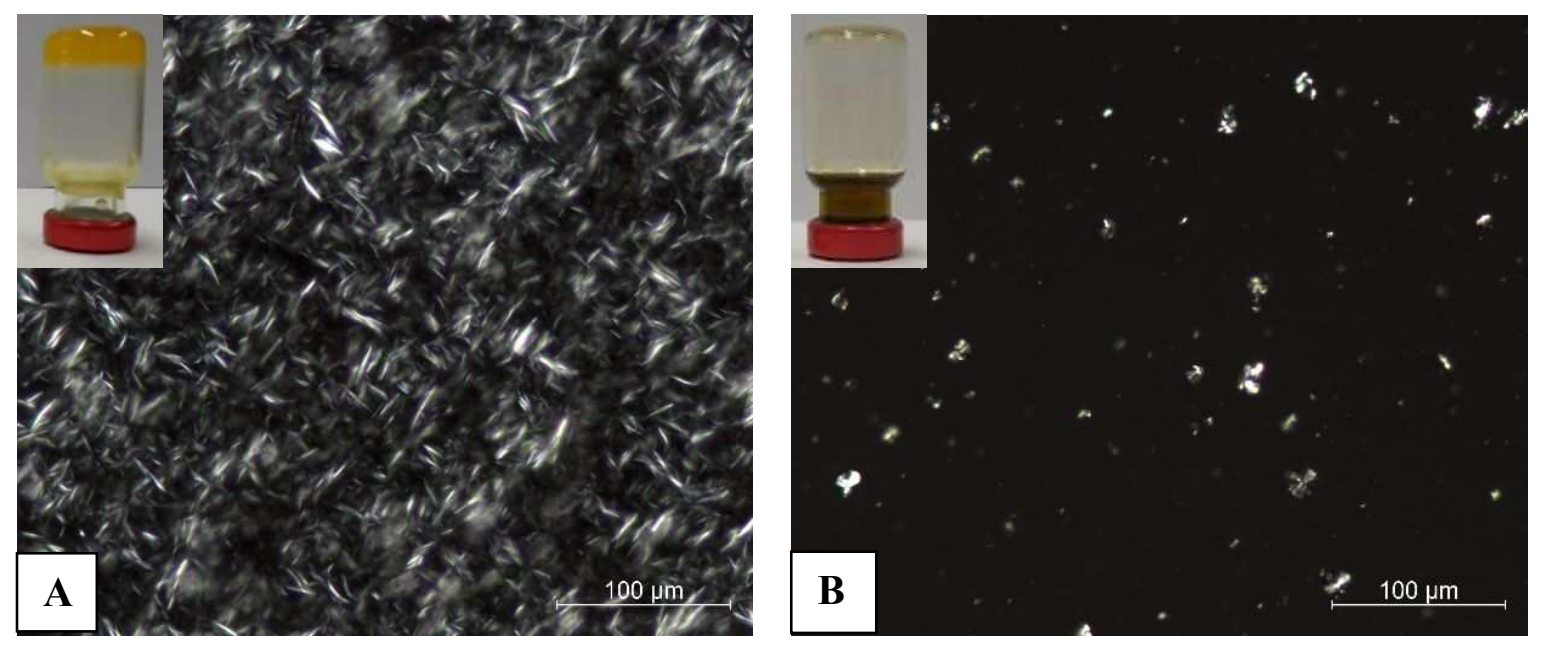

Fig 8 Polarized light microscopy pictures of linseed oil containing $5 \%$ w/w) (A) sunflower and (B) apple waxes produced with cooling rate of $6.0 \mathrm{~K} \mathrm{~min}^{-1}$. Inset: vials containing the samples turned upside down. 

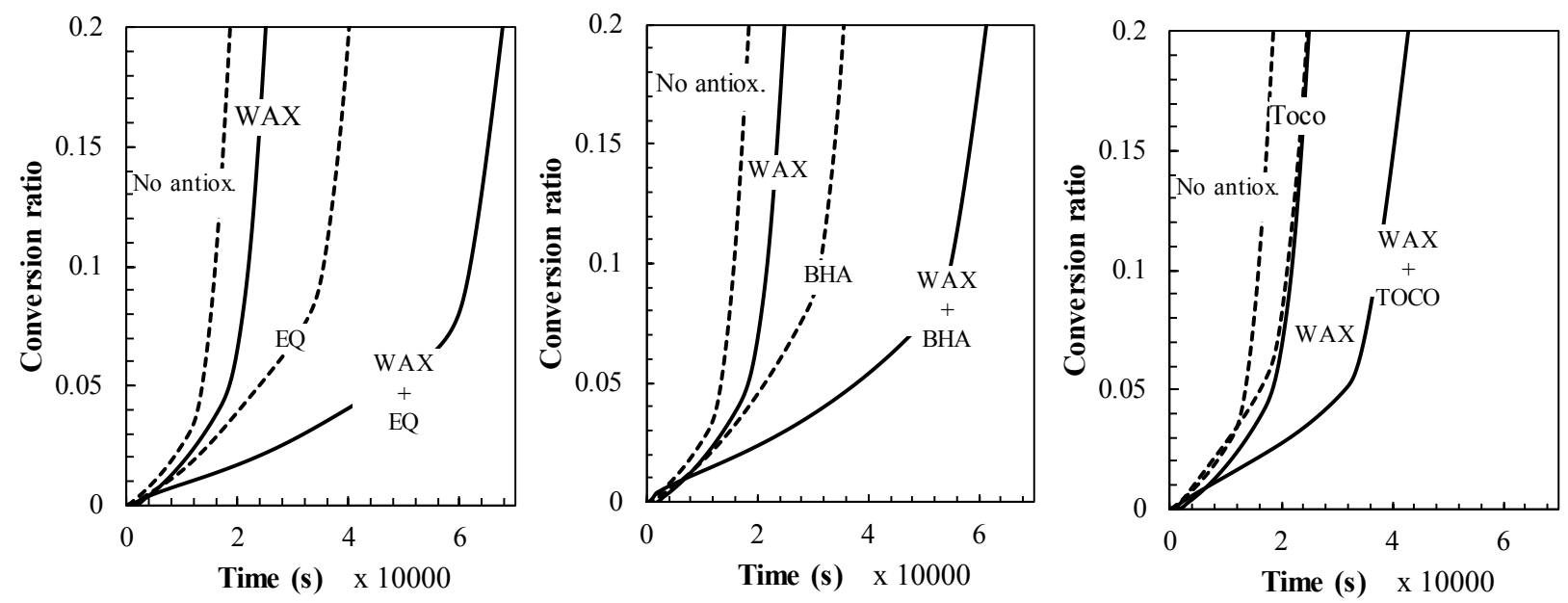

Fig 9 Conversion degree curves obtained by isothermal calorimetry of linseed oil without antioxidants, linseed oil with sunflower wax $(1 \%, w / w)$, linseed oil with BHA, ethoxyquin (EQ) or $\alpha$-tocopherol (TOCO) (1 mM), linseed oil with sunflower wax $(1 \%, \mathrm{w} / \mathrm{w})$ and BHA, ethoxyquin (EQ) or $\alpha$-tocopherol (TOCO) $(1 \mathrm{mM})$. The samples were prepared by mixing the oil with wax and antioxidant at $353 \mathrm{~K}$ for $20 \mathrm{~min}$, following cooling at controlled rate of $6.0 \mathrm{~K} \mathrm{~min}$. $^{-1}$ 


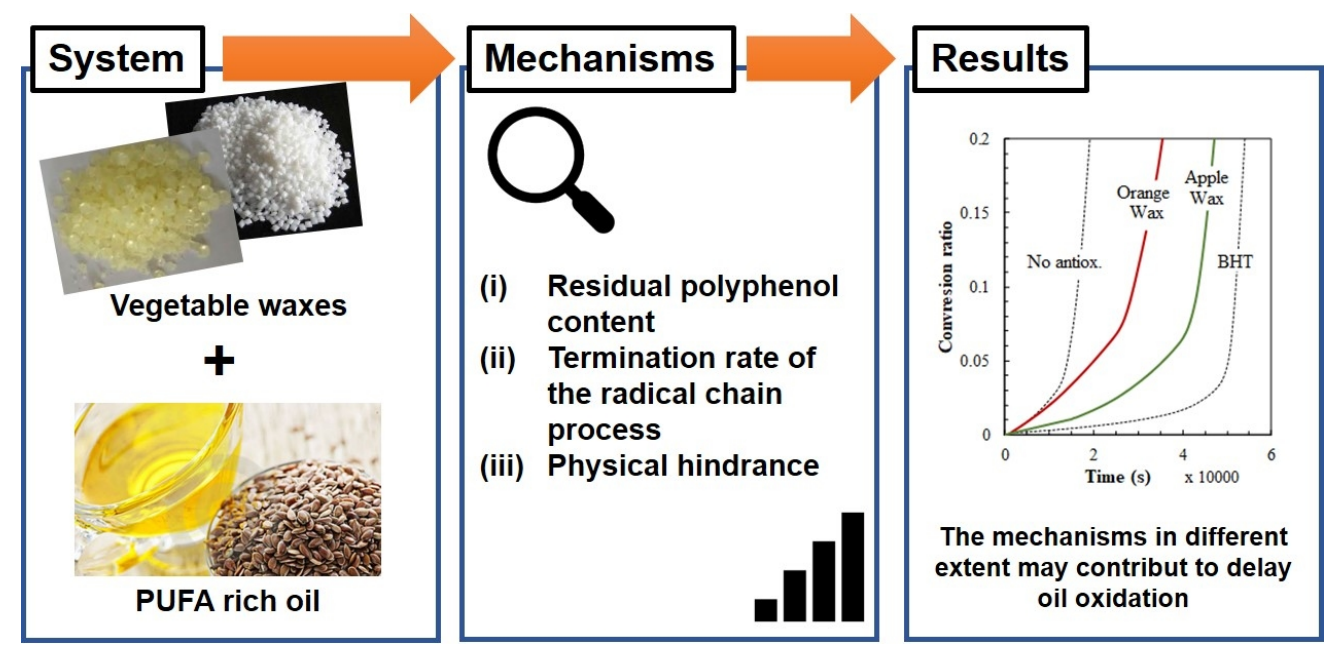

$214 \times 104 \mathrm{~mm}(150 \times 150 \mathrm{DPI})$ 